\title{
Two-dimensional finite element model of breast cancer cell motion through a microfluidic channel
}

Jared Barber · Luoding Zhu

Received: date /Accepted: date

\begin{abstract}
A two-dimensional model for red blood cell motion is adapted to consider the dynamics of breast cancer cells in a microfluidic channel. Adjusting parameters to make the membrane stiffer, as is the case with breast cancer cells compared with red blood cells, allows the model to produce reasonable estimates of breast cancer cell trajectories through the channel. In addition, the model produces estimates of quantities not as easily obtained from experiment such as velocity and stress field information throughout the fluid and on the cell membrane. This includes locations of maximum stress along the membrane wall. A sensitivity analysis shows that the model is capable of producing useful insights into various systems involving breast cancer cells. Current results suggest that dynamics taking place when cells are near other objects are most sensitive to membrane and cytoplasm elasticity, dynamics taking place when cells are not near other objects are most sensitive to cytoplasm viscosity, and dynamics are significantly affected by low membrane bending elasticity. These results suggest that continued calibration and application of this model can yield useful predictions in other similar systems.
\end{abstract}

Keywords breast cancer cell dynamics; viscoelastic elements

J. Barber

Department of Mathematical Sciences, Indiana University-Purdue University Indianapolis, Indianapolis, IN, USA

Tel.: 317-274-6936

Fax: 317-274-3460

E-mail: jarobarb@iupui.edu

L. Zhu

Department of Mathematical Sciences, Indiana University-Purdue University Indianapolis, Indianapolis, IN, USA

This is the author's manuscript of the article published in final edited form as:

Barber, J., \& Zhu, L. (2019). Two-dimensional Finite Element Model of Breast Cancer Cell Motion Through a Microfluidic Channel. Bulletin of Mathematical Biology, 81(4), 1238-1259. https://doi.org/10.1007/s11538-018-00557-x 


\section{Introduction}

Several techniques have been used to model the dynamics and mechanics of cells immersed in fluid. These include the immersed boundary (Crowl and Fogelson 2010; Dupin et al. 2007; Peskin 1977), lattice-Boltzmann (Crowl and Fogelson 2010; Dupin et al. 2007), dissipative particle dynamics (Blumers et al. 2017; Pivkin et al. 2017), finite element (Barber et al. 2008; Hoppe and Linsenmann 2012), and boundary element techniques (Peng et al. 2011; Pozrikidis 2005) (see (Freund 2014) for a review). Many of these studies have been focused on red blood cells (RBCs) because they are relatively simple to model, including just a viscoelastic membrane/surface and a denucleated homogeneous cytoplasm, and because they are usually immersed relatively well in fluid. Recently, however, there has been growing interest in using these techniques to study metastatic cancer cells (Lykov et al. 2017; Rejniak 2016).

There are at least two reasons for this growing interest. The first reason is that development has begun on microfluidic devices for use with cancer cells. Cancer cells tend to have different flexibility compared to their noncancerous counterparts (Suresh 2007) and lateral displacement devices (Djukic et al. 2015; Khodaee et al. 2016) and microchannels (Hu et al. 2016; TruongVo et al. 2017) can use these differences to help identify metastatic cells. The second reason is because of the important role of metastasis in cancer progression. Approximately $90 \%$ of all cancer-linked deaths are metastasis related (Tremblay et al. 2008). Major events of the metastatic cascade include entrance of circulating tumor cells (CTCs) into a vessel (intravasation), travel through vessels (translocation), and exit of CTCs out of the vessel into surrounding tissue (extravasation). The dynamics of CTCs during these events, where they are immersed relatively well in fluid, are particularly amenable to the previously mentioned numerical techniques (Rejniak 2016).

Mechanotransduction, in which forces on cells can cause cells to react, plays an important role in cancer. Cytoskeletal tension can affect the signaling pathways occurring in cancer cells in multiple settings (Jaalouk and Lammerding 2009). Matrix stiffness has been known to both instigate and mitigate proliferation, apoptosis, and angiogenesis, depending on the situation (Chin et al. 2016). In addition, stiffer cancer extra-cellular matrix can promote tumor invasion including the epithelial-mesenchymal transition (transition toward migration, invasion, and death resistance) and increased proliferation (Leight et al. 2012; Majeski and Yang 2016). At the same time, estimating forces in vivo can be difficult and/or costly which is why many have looked towards modeling to help estimate forces as cancer cells go through the various stages of metastasis.

The advantages of using a model to consider these microfluidic and in vivo scenarios are manifold. As in other similar situations, in silico experiments are often cheaper in terms of both time and money than their in vitro and in vivo counterparts. In addition, physical properties of cancer cells such as stiffness (Swaminathan et al. 2011) and adhesivity (Paredes et al. 2005; Ribeiro et al. 2010) can influence the cells' metastatic potential and physical forces can cause the previously discussed mechanotransduction. A mechanistic model can yield insight into physical properties of cancer cells and forces arising during movement which, in turn, can yield insight into metastatic potential and which cellular processes may be triggered in different scenarios.

The simulations resulting from the immersed boundary method of Rejniak (Rejniak 2012) suggested that optimal adherence of CTCs during extravasation can be obtained if the elasticity of the CTC cytoskeleton changes as time progresses. The dissipative particle dynamics model of Lykov (Lykov et al. 2017), which was designed for general eukaryotic cells, showed that cellular dynamics are significantly altered by the presence of a nucleus, slowed down by physiologically realistic increases in the stiffness of the cell's nuclear and cytoplasmic protein networks, and slowed down even more in the presence of viscous resistance in those protein networks.

Such models serve to demonstrate the potential usefulness of computational and mechanical modeling of immersed cells. At the same time, however, investigating details of implementation for these models alongside the $\mathrm{RBC}$ models suggests that each numerical technique has its strengths and weaknesses. More often than not there is a complex balance between computational efficiency, ease of implementation, model accuracy, and desired level of insight that must be considered in order to optimally investigate any one particular biological issue.

With an eye toward diversifying the set of tools available for computational modeling of CTCs, we have adapted another numerical technique for modeling RBCs (Barber et al. 2008; Barber et al. 2011; Secomb et al. 2007) for use with cancer cells. The technique's strengths include its efficiency when modeling a small number of cells (simulations typically take less than a day on a single processor), its inclusion of viscous forces in the cell, and its ability to resolve, to some degree, flow in narrow gaps between solids. The technique's weaknesses currently include difficulty with large numbers of cells and parallel implementation. The goal of this paper is to show that while this model is still being adjusted, it is another viable tool for considering circulating tumor cells in various scenarios.

The two-dimensional model is composed of a set of interconnected viscoelastic and viscous elements immersed in low Reynolds number flow. It has been adjusted to produce motion that agrees qualitatively well with 
the motion of typical epithelial and cancerous breast cells through a tapered microchannel (TruongVo et al. 2017). In addition, using the model alongside a sensitivity analysis allows consideration of how altered physical properties may affect cell dynamics. We believe that further model adaptations will allow consideration of dynamics of small numbers of CTCs in diagnostic microfluidic devices and in in vivo scenarios where CTCs travel in vessels and interact with vessel walls in three dimensions. This will help with the ultimate problem of obtaining insight into the mechanical forces occurring during CTC movement and the various cellular actions that may be mechanotransduced during that motion.

\section{Methods}

\subsection{Mathematical model}

The method, first presented in (Secomb et al. 2007) and (Barber 2009), consists of a single cell immersed in low Reynolds number flow. The cell is modeled using $n$ external nodes connected to one internal node, as pictured in Figure 1a. The motion of the flow and cell are coupled through the fluid and solid forces that meet at the cell's membrane, as seen in Equation 1 below. Because inertial forces are considered negligible, the forces throughout the entire system at any given moment must sum to zero. We present more details of the model below. Further details can be found in the previously mentioned manuscripts. The two-dimensional model is best thought of as representing a cross-section through the middle of a cell. In addition, grouped together, the cell acts as a Kelvin solid with viscous resistance to deformation and elastic energy to restore the cell to its original shape when displaced from that shape. Throughout what follows the term "membrane" is used to denote both the lipid bilayer and the protein structures anchored into it (cortex).

\subsubsection{Cell model}

Here we use $n=20$, unless otherwise noted, $L_{i}$ and $l_{i}$ are the lengths of the $i^{\text {th }}$ internal and external element, respectively, and $\alpha_{i}$ corresponds to the exterior angle between the $(i-1)^{\text {st }}$ and $i^{\text {th }}$ external elements (Figure 1a).
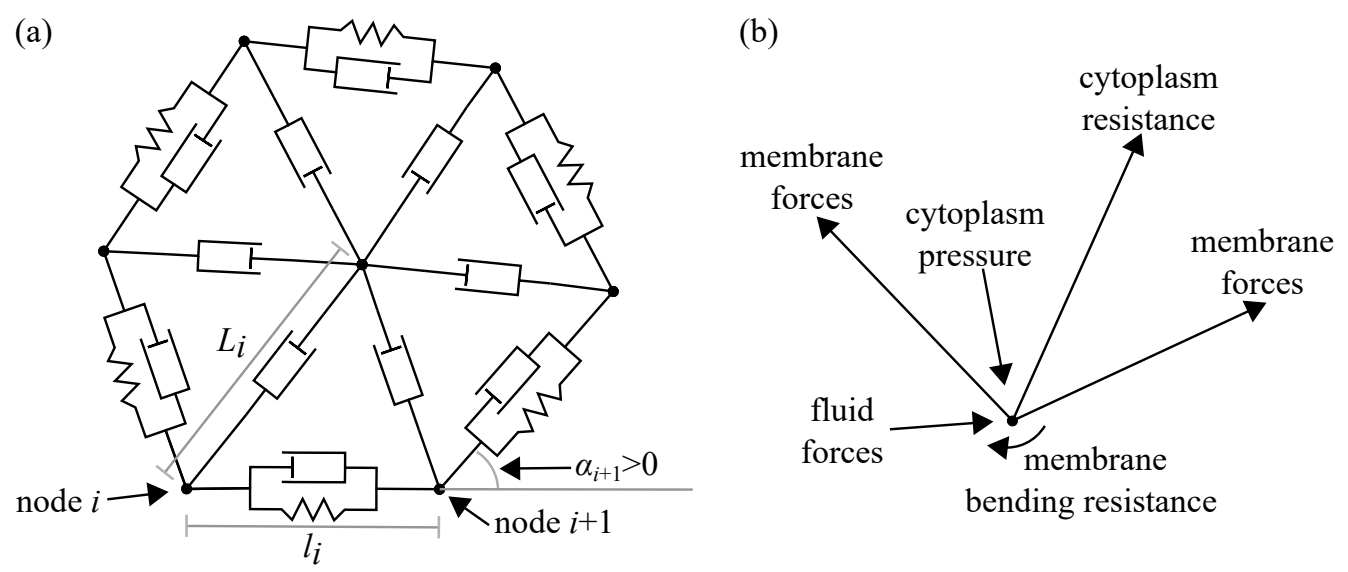

Figure 1: (a) Two-dimensional model representing a cross-section through a cell: interconnected external viscoelastic and internal viscous elements. The lengths of the $i^{\text {th }}$ internal and external elements are given by $L_{i}$ and $l_{i}$, respectively. $\alpha_{i}$ is the exterior angle at the $i^{\text {th }}$ node of the polygon formed by the external elements. (b) All stresses and moments that are considered when obtaining the nodal force balance in Equation 1 at a typical node $i$.

The mean tension, $\bar{t}_{i}$, in the $i^{\text {th }}$ external element due to the membrane is given by:

$$
\bar{t}_{i}=k_{t}\left(\frac{l_{i}}{l_{\text {ref }}}-1\right)+\frac{\mu_{\text {ext }}}{l_{i}} \frac{d l_{i}}{d t}
$$

where $k_{t}$ and $\mu_{\text {ext }}$ are the elastic and viscous coefficients for the viscoelastic external elements while $l_{r e f}$ is a reference length and $t$ is time. Parameter selection, description, and values can be found in Section 2.2 and Table 1. A bending moment is generated between external elements at node $i$ :

$$
m_{i}=-\frac{2 k_{b} \tan \left(\frac{\alpha_{i}}{2}\right)}{l_{\text {ref }}} .
$$


$k_{b}$ is the bending modulus and this nonlinear form has been chosen so that when $\alpha_{i}$ is small, $m_{i}=-\frac{k_{b} \alpha_{i}}{l_{r e f}}$, which is in agreement with earlier versions of this model (Barber et al. 2008), but when $\alpha_{i}$ approaches $\pm 180^{\circ}$, the bending moment becomes increasingly large. This keeps the membrane from forming physiologically unrealistic sharp corners. Note that for circular arcs, as the number of nodes go to infinity, this bending moment results in stresses consistent with those arising when using the Helfrich curvature free energy.

The cell interior is represented by internal elements and an interior pressure. The tension in the $i^{\text {th }}$ internal element due to the membrane, $T_{i}$, is given by:

$$
T_{i}=\frac{\mu_{i n t}}{L_{i}} \frac{d L_{i}}{d t}
$$

where $\mu_{\text {int }}$ is the viscosity of the linearly viscous internal elements. The interior pressure is given by:

$$
p_{\text {int }}=k_{p}\left(1-A / A_{\text {ref }}\right)
$$

where $k_{p}$ is an area enforcement constant, $A$ is the area of the cell, and $A_{\text {ref }}$ is a reference area. The larger $k_{p}$ is, the closer the cell's area will be to the reference area throughout the simulation.

\subsubsection{Fluid model}

Since the Reynolds number is small, around 0.09, and the motion of fluid is quite slow, the steady state Stokes flow equations are used to model the fluid flow. The conservation of momentum equation is given by:

$$
\nabla \cdot \boldsymbol{\sigma}=0 ; \quad \boldsymbol{\sigma}=\mu\left(\nabla \boldsymbol{u}+\nabla \boldsymbol{u}^{T}\right)-p \boldsymbol{I} .
$$

$\boldsymbol{\sigma}$ is the fluid stress tensor, $\mu$ is the dynamic viscosity, $\boldsymbol{u}=(u, v)^{T}$ is the fluid velocity vector, and $p$ is the pressure in the fluid. Continuity is enforced using the equation

$$
\nabla^{2} p=K \nabla \cdot \boldsymbol{u}
$$

Using $K=10^{8}$ dyn s/cm $\mathrm{cm}^{4}$ helps maintain reasonable levels of incompressibility in simulations (Secomb et al. 2007).

\subsubsection{Coupling models via nodal equations}

We denote the unit vectors that are normal and tangential to the $i^{\text {th }}$ external element as $\boldsymbol{n}_{i}$ (pointing away from the cell interior) and $\boldsymbol{t}_{i}$ (pointing counterclockwise around the cell), respectively. The unit vector tangential to the $i^{\text {th }}$ internal element is denoted by $\boldsymbol{T}_{i}$ (pointing inwards). The normal and tangential components of the non-membrane related stresses are given by $f_{i}=\boldsymbol{n}_{i}^{T} \boldsymbol{\sigma} \boldsymbol{n}_{i}+p_{\text {int }}$ and $g_{i}=\boldsymbol{t}_{i}^{T} \boldsymbol{\sigma} \boldsymbol{n}_{i}$. Positive $f_{i}$ forces the membrane outward while positive $g_{i}$ pulls the membrane counterclockwise.

Membrane mechanics (Evans and Skalak 1979) relates mean shear stress in an external element, $\bar{q}_{i}$, to the bending moments at either end of the element in the following way:

$$
\bar{q}_{i}=\frac{1}{l_{i}} \int_{0}^{l_{i}} \frac{d m_{i}(s)}{d s} d s=\frac{m_{i}\left(l_{i}\right)-m_{i}(0)}{l_{i}}=\frac{-2 k_{b}\left(\tan \left(\frac{\alpha_{i+1}}{2}\right)-\tan \left(\frac{\alpha_{i}}{2}\right)\right)}{l_{\text {ref }} l_{i}} .
$$

Here $s$ is the arc length along the $i^{\text {th }}$ external element with $s=0$ at node $i$ and $s=l_{i}$ at node $i+1 . m_{i}(s)$ is the bending moment as a function of arclength along the $i^{\text {th }}$ external element. Further relations from membrane mechanics can be used to see that tension, $t_{i}(s)$, and shear stress, $q_{i}(s)$, along the element can be found using the mean tension and shear stresses:

$$
t_{i}(s)=\bar{t}_{i}+\frac{1}{l_{i}} \int_{0}^{l_{i}}\left(s-s^{\prime}\right) g_{i}\left(s^{\prime}\right) d s^{\prime} ; \quad q_{i}(s)=\bar{q}_{i}+\frac{1}{l_{i}} \int_{0}^{l_{i}}\left(s-s^{\prime}\right) f_{i}\left(s^{\prime}\right) d s^{\prime} .
$$

Summing up all shearing and tensional stresses acting at the $i^{\text {th }}$ external node (Figure $1 \mathrm{~b}$ ) gives:

$$
t_{i}(0) \boldsymbol{t}_{i}-t_{i-1}\left(l_{i-1}\right) \boldsymbol{t}_{i-1}-q_{i}(0) \boldsymbol{n}_{i}+q_{i-1}\left(l_{i-1}\right) \boldsymbol{n}_{i-1}+T_{i} \boldsymbol{T}_{i}=0 \text {. }
$$

Summing up all forces arising from the internal viscous elements at the central node yields:

$$
\sum_{i=1}^{n} T_{i} \boldsymbol{T}_{i}=0
$$

These two nodal equations include unknown velocities of the nodes on the cell (nodal velocities) that come from the viscous terms in the external and internal tensions and are coupled with the Stokes equations through the fluid stresses acting on the cell, $f_{i}$ and $g_{i}$. These stresses are, in turn, dependent on the fluid stress tensor $\boldsymbol{\sigma}$. This formulation is, therefore, a fully coupled fluid-structure-interaction (FSI) method.

\subsubsection{Lubrication corrections}

While finite element solvers (such as FlexPDE 7) often use adaptive meshing to more effectively resolve problems with multiple scales, this approach has its limits as matrix conditioning can become worse as the finite elements 
used span a larger range of sizes. In this case, the issue manifests itself in regions where narrow gaps, which require small finite elements, form between two solid surfaces (e.g. Figure 3c). Combining this issue with the fact that the cell's membrane, which is physiologically relatively smooth, is represented by a cornered polygon leads the finite element method to perform suboptimally in narrow gap regions between corners and walls. While the method still tends to outperform most naïve methods for membrane dynamics, adding additional model components can further improve performance in narrow gap regions.

To address this, an additional term, $\boldsymbol{f}_{\text {gap }}$, is added to the above force equation when cell nodes become close to microchannel walls and when cell walls become close to microchannel corners. The term used here is very similar to a relatively well-established approach involving linear elastic terms (Dong and Skalak 1992; Drury and Dembo 2001; Leong et al. 2011; Marella and Udaykumar 2004). In this model,

$$
\boldsymbol{f}_{\text {gap }}=-\frac{c_{r}}{n} \frac{\left(\varepsilon-h_{l}\right)^{2}}{\varepsilon h_{l}} \boldsymbol{n}_{w} \text {. }
$$

$c_{r}$ is a "repulsive force" coefficient, $h_{l}$ is the distance from the corner to the wall, and $\boldsymbol{n}_{w}$ is a unit vector normal to the wall and pointing towards the corner. $\varepsilon$ is a threshold value for the corner-wall distance and $\boldsymbol{f}_{\text {gap }}$ is calculated/included only when $h_{l}$ drops below $\varepsilon$. The functional form was chosen in order to guarantee that gap thickness does not converge to zero (as $\left.h_{l} \rightarrow 0, \boldsymbol{f}_{\text {gap }} \propto 1 / h_{l}\right)$ and to eliminate possibly abrupt introduction of these forces as $h_{l}$ switches from $h_{l} \geq \varepsilon$ to $h_{l}<\varepsilon$. The latter is accomplished because

$$
\left.\frac{d \boldsymbol{f}_{\text {gap }}}{d h_{l}}\right|_{h_{l}=\varepsilon}=\mathbf{0},
$$

and this choice significantly improves the associated time stepping when narrow gaps form. More details on how various practical issues are dealt with when applying such additional gap forces can be found in (Barber 2009).

The motivation for this term is that the cell surface and microchannel wall tend to exhibit surface roughness so that when the gap is small enough solid-solid interactions take place that inhibit the corner from moving more closely towards the wall. While previous versions of this model used more sophisticated lubrication terms and other interventions (Barber 2009), here a less sophisticated model is employed because simulations suggest little difference between the two approaches with the exception that the more sophisticated model can fail for small enough gaps.

\subsubsection{System solution and integration}

The nodal equations are coupled to the finite element equations for Stokes flow (see 2.3.1 for boundary conditions) and all velocities are simultaneously solved for using FlexPDE (version 7.0.9), including nodal velocities, $\boldsymbol{u}_{i}^{j}$, where $i$ corresponds to the $i^{\text {th }}$ node and $j$ corresponds to the current $j^{\text {th }}$ time step. The finite element solver uses cubic elements, making each solve $3^{\text {rd }}$ order accurate in space, and Lanczos iteration (Jea and Young 1983) for solving the underlying stiffness matrix equation. It also uses adaptive meshing based on a posteriori error estimates (PDE Solutions Inc 2018) to further improve accuracy (see Figure 3c). Resulting estimated local relative errors are less than 1e-3 (the error tolerance that was chosen for these simulations).

After each spatial solve, the obtained $\boldsymbol{u}_{i}^{j}$ are used in an adaptive time-stepping scheme in order to solve the ordinary differential equations associated with the positions of the nodes: $\frac{d x_{i}}{d t}=\boldsymbol{u}_{i}$. The method is second order accurate and gives the positions of the nodes at the next time, $\boldsymbol{x}_{i}^{j+1}$ :

$$
\boldsymbol{x}_{i}^{j+1}=\boldsymbol{x}_{i}^{j}+\left(\tau_{j+1}-\tau_{j}\right)\left(\boldsymbol{u}_{i}^{j}+\frac{\tau_{j+1}-\tau_{j}}{2\left(\tau_{j}-\tau_{j-1}\right)}\left(\boldsymbol{u}_{i}^{j}-\boldsymbol{u}_{i}^{j-1}\right)\right)
$$

Here $\tau_{j}$ corresponds to the time at the $j^{\text {th }}$ time step. When uniform time stepping is used, the scheme becomes the traditional second order Adams-Bashforth method. In order to maintain stability, the method requires slightly more functional evaluations per simulation than forward Euler (Secomb et al. 2007) and Heun's method (Barber et al. 2011). Nonetheless, the new method is appealing because of the relative ease with which adaptive time-stepping is implemented, its $2^{\text {nd }}$ order accuracy, and its relative robustness when working together with the finite element iterative solver. By limiting the time step so that the relative change in nodal velocities was less than $1 \%$ per step, reasonable simulations were obtained. 


\subsection{Parameter selection}

A cell diameter of $D_{r e f}=12.4 \mu \mathrm{m}$ was used, which agrees with the cancer cells (MDA-MB-231) from (TruongVo et al. 2017). The roundness of a given shape is defined as the area of the shape divided by the area of a circle with the same perimeter as the shape. Red blood cells have a biconcave shape and the roundness of their cross-sections tend to be significantly less than 1. In line with (Hu et al. 2016; TruongVo et al. 2017), however, we have assumed roughly spherical breast cancer cells at rest which corresponds to approximately circular cross-sections where the roundness is approximately 1 . This gives $A_{\text {ref }}=\pi D_{\text {ref }}^{2} / 4$ and $l_{\text {ref }}=\pi D_{\text {ref }} / n$.

Early simulations using material parameters from the red blood cell model showed a tendency for cell membranes to fold in such a way that $\alpha_{i} \approx \pm 180^{\circ}$. While for a soft enough material, this might be reasonable (for example, see (Secomb 1987) for cusps on bending resistance-free membranes), shapes seen in (Hu et al. 2016; TruongVo et al. 2017) suggest such folding is unlikely for these cells. With this in mind and the fact that red blood cells tend to be significantly more flexible than their nucleated counterparts (Freund 2014), we multiplied $k_{t}$ and $k_{b}$ by 100 . This produced more realistic results with less drastic folding.

In addition, we found that cells tended to elongate more than experiments might suggest. Adjusting $k_{p}$ from 50 to 100 seemed to be most effective in reducing the length of the simulated cells, which corresponds to keeping the cross-sectional area closer to its reference value. Assuming volume conservation of the experimental cells and that such cells take on the shape of a truncated sphere as they pass through the device, as done in (Hu et al. 2016; TruongVo et al. 2017), one can estimate that the cross-sectional area of $12.4 \mu \mathrm{m}$ diameter cells should be reduced to $66 \%$ of their initial cross-sectional area as they passes through the microfluidic channel. For our model, $k_{p}=50$ led to the area of the cell being reduced to approximately $63 \%$ of its original area while $k_{p}=100$ corresponded to $70 \%$ reduction. To avoid getting farther from the target area reduction value of $66 \%$, we did not increase $k_{p}$ further.

While we do not show these results, we add that we also explored enforcing $100 \%$ area conservation throughout simulations with results that differed significantly more from experiment than the ones shown here. We also considered enforcing volume conservation by adjusting the interior pressure so that an estimate of the cell's volume (obtained by assuming the cell is approximately axisymmetric along a particular axis) is held approximately constant. This produced results similar to the ones shown here suggesting that the added level of complexity was not needed.

The other parameters were unchanged from (Secomb et al. 2007) and can be found in Table 1.

\begin{tabular}{|c|c|c|}
\hline Parameter & Description & Value \\
\hline$l_{r e f}$ & Elastic reference length for external elements & $1.96 \mu \mathrm{m}$ \\
\hline$A_{r e f}$ & Elastic reference area for cell & $120.76 \mu \mathrm{m}^{2}$ \\
\hline$k_{t}$ & Elastic coefficient for external elements & $1.2 \mathrm{dyn} / \mathrm{cm}^{-1}$ \\
\hline$k_{b}$ & Bending modulus for junctures between external elements & $9 \times 10^{-10} \mathrm{dyn} \mathrm{cm}$ \\
\hline$k_{p}$ & Constant for keeping area near $A_{r e f}$ & $1000 \mathrm{dyn} / \mathrm{cm}^{2}$ \\
\hline$\mu_{\text {ext }}$ & Viscosity coefficient for external elements & $2 \times 10^{-4} \mathrm{dyn} \mathrm{s} / \mathrm{cm}$ \\
\hline$\mu_{i n t}$ & Viscosity coefficient for internal elements & $1 \times 10^{-4} \mathrm{dyn} \mathrm{s} / \mathrm{cm}^{-2}$ \\
\hline$\mu$ & Viscosity of surrounding fluid & $10^{-2} \mathrm{dyn} \mathrm{s} / \mathrm{cm}^{2}$ \\
\hline$K$ & Penalty constant for enforcing incompressibility & $10^{8} \mathrm{dyn} \mathrm{s} / \mathrm{cm}^{4}$ \\
\hline$\varepsilon$ & Threshold above which no lubrication terms are added & $10^{-2} \mu \mathrm{m}$ \\
\hline$c_{r}$ & Repulsive force constant for solid-solid interactions & $5 \times 10^{-4} \mathrm{dyn}$ \\
\hline
\end{tabular}

Table 1: Baseline parameter values used for the simulations.

\subsection{Simulations}

The simulations have been designed to match the breast cancer cells (MDA-MB-231) traveling through the thinner microchannels of the higher speed experiments found in (TruongVo et al. 2017). This includes the geometry of the domain pictured in Figure 2, a cell diameter of $12.4 \mu \mathrm{m}$, the assumption of an approximately spherical cell, and the average velocities of 3.5 and $11 \mathrm{~mm} / \mathrm{s}$ at the inlet and outlet, respectively. This particular microchannel device was designed as a possible way to sort breast cancer cells. Breast cancer cells tend to be more flexible than regular 
breast cells and, as such, tend to make it through the microchannels more easily. The leaders of this study were hoping that such microchannels could be used to more quickly identify CTCs in patients.

\subsubsection{Boundary and initial conditions}

Figure 2 shows the computational domain (grayed in) and boundaries. A no-slip condition is used on the stationary microchannel wall (black boundary lines) and moving membrane wall (solid blue lines). At the left and right ends flow is assumed to be relatively well developed with $v=0$ and $\frac{\partial u}{\partial \boldsymbol{n}}=0$ (normal derivative; dashed green lines). Pressures are also assigned at those ends so that 1 ) the pressure drop produces flow velocities of approximately 3.5 and $11 \mathrm{~mm} / \mathrm{s}$ at the inlet and outlet, respectively, when no cells are present and 2) the average fluid pressure, $p$,on the cell's membrane is approximately 0 :

$$
\frac{\oint p d \Omega_{\text {membrane }}}{\oint d \Omega_{\text {membrane }}}=0 .
$$

This latter constraint determines the arbitrary fluid pressure constant and makes the pressure unique. The horizontal boundaries that lie within $40 \mu \mathrm{m}$ of the left and right ends (red dashed lines) are open and invoking approximate symmetry we set $v=0$ and $\frac{\partial u}{\partial \boldsymbol{n}}=0$ at those boundaries. Using a Laplacian-based penalty method for the continuity equation requires prescribing additional boundary conditions for the pressure. We set $\frac{\partial p}{\partial \boldsymbol{n}}=0$ at all but the left and right boundaries (dashed green lines). For the large value of $K$ chosen, results do not appear to depend significantly on these additional pressure boundary conditions.

Only one cell is modeled in any given simulation. It starts at $-170 \mu \mathrm{m}$ to the left of the origin and is simulated until it is within $0.1 \mu \mathrm{m}$ of the rightmost boundary. Because dynamics in the narrow outlet region is of primary interest, this is where cells experience their highest levels of deformation and deformation rates, it was decided that starting with an unbiassed circular shape would be reasonable as it should not significantly affect outlet region dynamics.

\subsubsection{Typical baseline dynamics}

Using the baseline parameters (Table 1) we run a simulation to obtain typical cell trajectories and use the finite element method to investigate the velocity and stress fields when the cell is in the narrow outlet region (Figure 3 and Figure 4).

\subsubsection{Parameter sensitivity analysis}

To show that the model can be used to investigate the sensitivity of cell dynamics to model parameters, we perform a sensitivity analysis using the material parameters $k_{t}, k_{b}, \mu_{\text {int }}$, and $\mu_{\text {ext }}$ around the baseline values in Table 1 . In particular, for each parameter, we investigate sensitivity by running simulations for 0.5 and 2 times the baseline values for that parameter. For instance, for $k_{t}$ all parameters are held at their baseline values except $k_{t}$ which is changed to $0.6 \mathrm{dyn} / \mathrm{cm}$ for one simulation and $2.4 \mathrm{dyn} / \mathrm{cm}$ for another simulation. The results of these two simulations are then compared with baseline in order to estimate any trends in behavior as the parameter is changed. No more than one parameter was changed at a time.

Because of the adaptive time stepping used, the discrete times at which cell shapes are available depend on the simulation. To assist comparison, the geometric centroids of the cells were calculated for each shape obtained during the time-stepping procedure. Linear interpolation with respect to the $x$-coordinates of the centroids $\left(x_{c}\right)$ was then implemented in order to obtain estimates of the shapes of the cells for non-baseline simulations at the centroid locations available for the baseline simulation. The agreement between two simulations for any given centroid location can then be quantified using

$$
\% \operatorname{Agreement}\left(x_{c}\right)=\frac{\text { area inside both baseline and non-baseline cell shapes (overlap area) }}{((\text { area of the baseline shape })+(\text { area of the non-baseline shape })) / 2} \text {. }
$$

This measure compares the shapes of two cells when the $x$-coordinates of their centroids both lay at $x=x_{c} \mu \mathrm{m}$, is purely a function of the geometry of the two cells for a fixed $x_{c}$, and does not depend explicitly on the amount of time for which the cells have travelled. Higher values of $\%$ Agreement correspond to more agreement and less sensitivity of the model to the parameter change that was made. \% Agreement is a function of $x_{c}$ but for simplicity, only the minimum \% Agreement is included in Table 2. Percentage change in passage times (defined as the time it takes for the cell to travel from -170 to $170 \mu \mathrm{m}$ ) are also included in comparisons. Comparison plots include cell shapes that have the exact same centroid locations and the centroid locations chosen for the plots approximately minimize the \% Agreement (maximize shape disagreement) between cell shapes. 


\section{Results}

\subsection{Baseline simulation}

A typical trajectory for a cell for the baseline parameter values used can be seen in Figure 2 which shows cell membrane positions at $0,4, \ldots, 48 \mathrm{~ms}$. For comparison, it takes approximately $40.4 \mathrm{~ms}$ for the cell to travel from 150 to $150 \mu \mathrm{m}$ while, on average, it takes fluid particles $41 \mathrm{~ms}(300 \mu \mathrm{m} /((3.5 \mu \mathrm{m} / \mathrm{ms}+11 \mu \mathrm{m} / \mathrm{ms}) / 2)$ to travel the same distance.

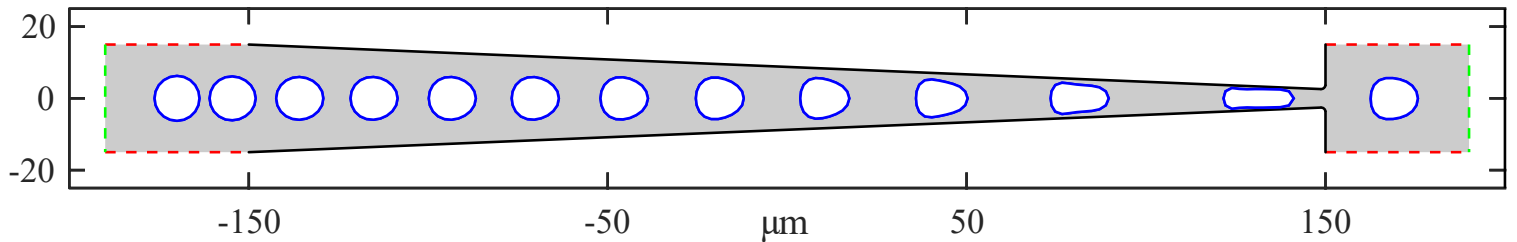

Figure 2: Trajectory of a cell using baseline parameter values and domain used for simulations. Includes cell membrane position every $4 \mathrm{~ms}$ and the entire shown simulation lasts approximately $52 \mathrm{~ms}$.

There are two major competing effects worth noting when discussing this result. Because the cell is in the center of the channel, it lies in the center of the approximate Poiseuille (parabolic) flow profile, which corresponds to the highest velocity flow across the channel. This causes the cell to move faster than the average fluid velocity in the region. At the same time, as the cell travels through the channel, it raises the effective flow resistance of the channel causing general motion through the channel to become slower. These effects seem to cancel out producing nearly identical travel times for the cell vs an average fluid particle.

One of the benefits of using a model is that it can produce results that are not as easily obtained from experiment. For instance, Figure 3 shows estimates of fluid speed throughout the system. In contrast, the velocity measurements in similar experiments are usually limited to average flow rates throughout the entire channel with more detailed velocity information requiring tracer particles and tracking software. While we are focused more on stresses in this study, knowing such velocities can help understand drug or other chemical deposition rates.

Figure $3 \mathrm{~b}$ shows that the flow far away from the cell does look approximately parabolic, which is in reasonable agreement with the approximate Poiseuille flow profile that should result in the absence of a cell. These plots, however, do illustrate typical expected dynamics with the cell looking like a water balloon attempting to squeeze through a narrow opening. Its front end bulges while its tail end collapses as high pressure from behind pushes on the rear end of the cell while low pressure at the cell's head pulls that portion of the cell outwards. The result is what looks like inflation of the front of the cell and deflation of the tail of the cell. This effect is further enhanced by the fact that when the cell membrane gets close to the microchannel corners, the thin fluid regions significantly slow down the progression of the cell's membrane through the gap. The result is that the membrane components on the front end stretch out while the membrane at the tail end effectively contract. This can result in physical instabilities in terms of membrane dynamics. The majority of the simulations presented here are approximately symmetric throughout the entire simulation but in one of our simulations, this crumpling effect led to asymmetric membrane dynamics in Figure $5 \mathrm{~b}$. 


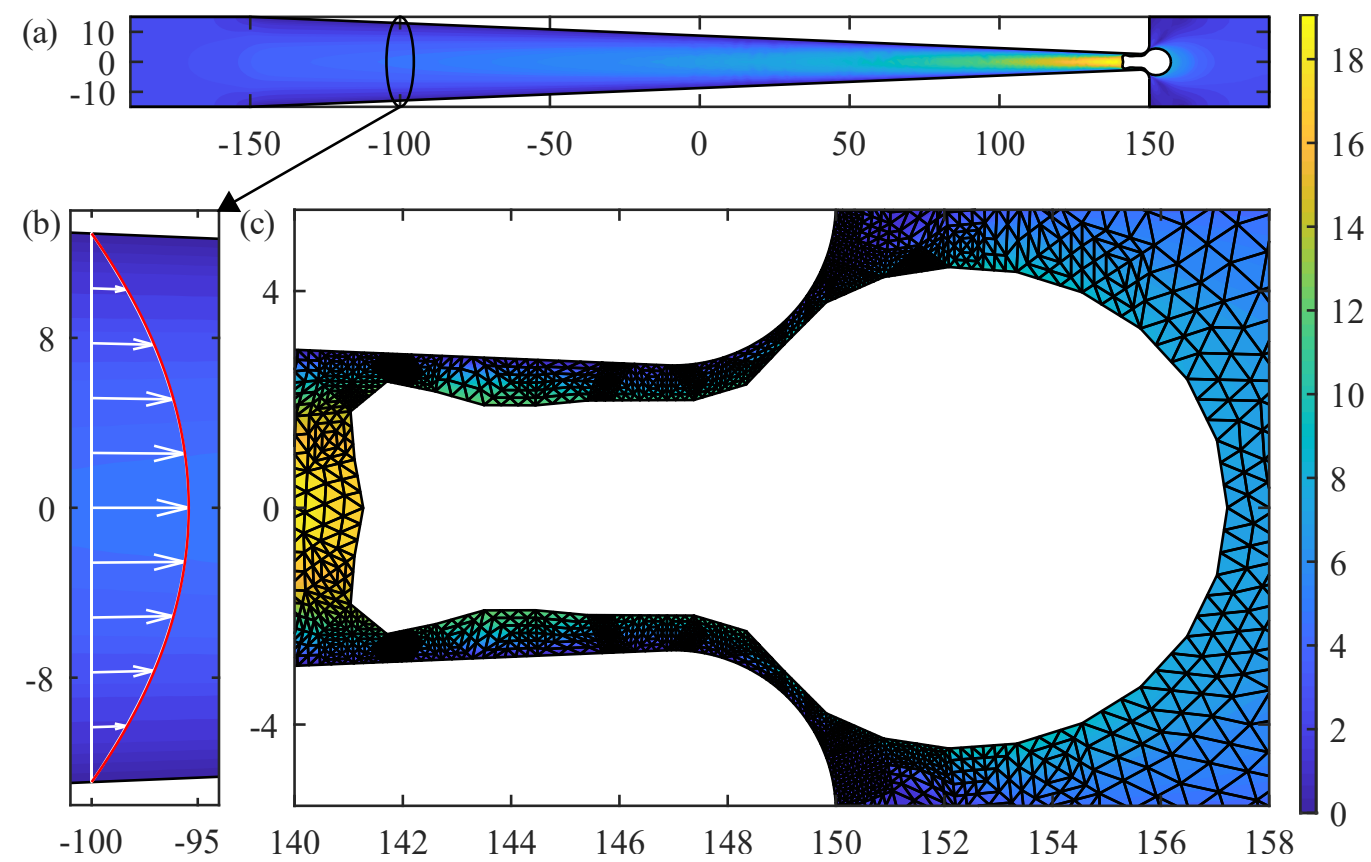

Figure 3: Units are in microns and microns/millisecond and 40 nodes were used for this simulation. (a) Speed of the fluid surrounding the cell plotted throughout the entire computational domain. (b) Velocity of the fluid far upstream from the narrow gap region. The red line is a parabola and was included to show that the flow profile is, in fact, very close to parabolic upstream of the narrow gap. (c) Zoomed in plot of the same speed with the corresponding finite element mesh shown. Adaptive meshing was used so that more finite elements can be found near the cell.

As previously mentioned, forces and stress levels in cancer cells may yield insight into mechanotransduction and overall cancer cell dynamics. The model is able to predict stress levels in the fluid and along the membrane. For instance, Figure $4 \mathrm{a}$ and $\mathrm{b}$ show the maximum of the absolute values of the principal stresses $\left(\sigma_{1,2}=\frac{\sigma_{11}+\sigma_{22}}{2} \pm\right.$ $\left.\sqrt{\left(\frac{\sigma_{11}-\sigma_{22}}{2}\right)^{2}+\sigma_{12}^{2}}\right)$ throughout the fluid. It can be seen that largest stresses arise in the narrow region between the cell and the corner of the microchannel. Figure $4 \mathrm{c}$ and d show the normal (c) and tangential (d) stresses along the membrane. These are the forces due to the fluid and, again, are highest in the narrow region between the cell and microchannel corner. The levels presented here are likely overestimates because in physiologically realistic systems sharp corners do not exist. Even in microchannels slight rounding can be seen at manufactured corners (TruongVo et al. 2017). Lack of sharp corners is even more pronounced in in vivo systems. Nonetheless, it makes sense that higher stress levels would be colocalized with the narrow gap regions where shear rates tend to be the highest. 

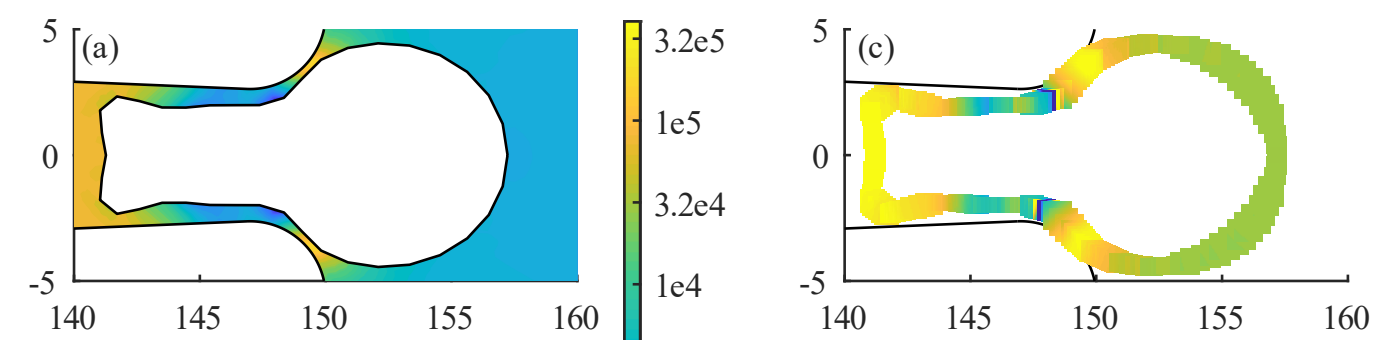

$1 \mathrm{e} 3$

$1 \mathrm{e} 2$

le1

$1 \mathrm{e} 0$

$3.2 \mathrm{e} 3$
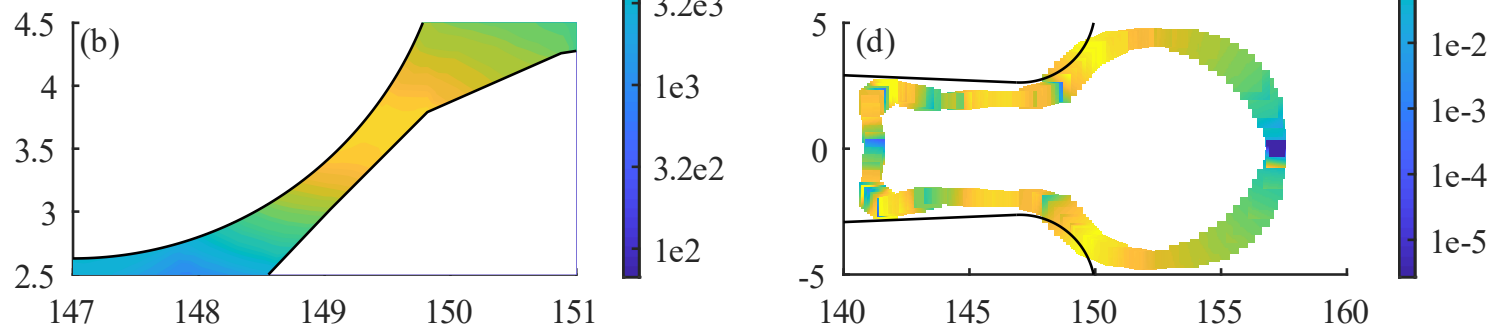

Figure 4: Units are in pascals for stress and microns for lengths and 40 nodes were used for these simulations. Upper left-maximum of the two principal stress magnitudes is shown in the fluid near the cell. Lower left-zoomed in plot showing the same maximum of the two principal stress magnitudes in the fluid near the microchannel corner. Upper right-normal stress on the cell membrane. Lower right-tangential stress on the cell membrane.

\subsection{Convergence analysis}

Cells were simulated for $1 \mathrm{~ms}$ in a tube with a width of $17.5 \mu \mathrm{m}$ and average flow speed of $7.25 \mu \mathrm{m} / \mathrm{ms}$ (same as the average width and flow speed for the microchannel). Boundary conditions were chosen to reproduce Poiseuille flow in the absence of a cell. For a second order method the \% Agreement between approximate cell shapes and the actual cell shape (corresponding to an infinite number of nodes) should go to $100 \%$ in a quadratic fashion. In particular, 100-(\% Agreement) should be $O\left(l_{i}^{2}\right)$. Assuming the cell shape resulting when the number of nodes is 80 is close to exact we see that $100-(\%$ Agreement) after $1 \mathrm{~ms}$ is approximately $2.03 \%$ for 10 nodes, $0.40 \%$ for 20 nodes and $0.087 \%$ for 40 nodes. This suggests that the method is at least second order in space and time, which agrees with theory.

\subsection{Sensitivity Analysis}

Models can also be used to investigate the relative influence of different parameters using a sensitivity analysis. The table below shows the relative changes of two model outputs when parameters are changed. Primed parameters correspond to the new parameters used in the corresponding simulations while unprimed parameters correspond to the values used in the original baseline simulations.

\begin{tabular}{|l|c|c|}
\hline & $\begin{array}{c}\text { \% Change total } \\
\text { passage time }\end{array}$ & $\begin{array}{c}\text { Minimum \% } \\
\text { Agreement }\end{array}$ \\
\hline$k_{p}^{\prime}=k_{p} / 2$ & $-1.1 \%$ & $94.6 \%$ \\
\hline$k_{p}^{\prime}=2 k_{p}$ & $1.2 \%$ & $94.6 \%$ \\
\hline$k_{b}^{\prime}=k_{b} / 2$ & $-0.01 \%$ & $68.3 \%$ \\
\hline$k_{b}^{\prime}=2 k_{b}$ & $0.5 \%$ & $98.1 \%$ \\
\hline$k_{t}^{\prime}=k_{t} / 2$ & $-0.2 \%$ & $93.1 \%$ \\
\hline$k_{t}^{\prime}=2 k_{t}$ & $0.2 \%$ & $94.8 \%$ \\
\hline$\mu_{\text {int }}^{\prime}=\mu_{\text {int }} / 2$ & $0.3 \%$ & $86.1 \%$ \\
\hline$\mu_{\text {int }}^{\prime}=2 \mu_{\text {int }}$ & $0.1 \%$ & $86.4 \%$ \\
\hline$\mu_{\text {ext }}^{\prime}=\mu_{\text {ext }} / 2$ & $-0.01 \%$ & $99.1 \%$ \\
\hline$\mu_{\text {ext }}^{\prime}=2 \mu_{\text {ext }}$ & $0.06 \%$ & $98.7 \%$ \\
\hline
\end{tabular}

Table 2: Sensitivity results including measures of differences between baseline and simulations when individual parameters are changed. Measures include the relative percentage change in the time it takes for cells to pass from 170 to $170 \mu \mathrm{m}$ (as seen in Figure 2) and the minimum \% agreement experienced over the entire simulation as defined by Equation 2 . 
In addition, Figure 5 shows comparisons between cell shapes when different parameters are changed. The shapes shown in this figure have been chosen so that the values of $x_{c}$ (x-values for the centroids) minimize the \% Agreement (maximize disagreement) between shapes. Comparison plots are included for all five comparisons involving $k_{p}, k_{b}, k_{t}, \mu_{\text {int }}$, and $\mu_{\text {ext }}$. These results suggest the following and are further supported by similar plots not shown here. Significant cell shape changes result when the area parameter, $k_{p}$, bending elasticity, $k_{b}$, external element (elongational) elasticity, $k_{t}$, and internal element viscosities, $\mu_{i n t}$, are varied, particularly when the cell moves through the narrow outlet $\left(k_{p}\right.$ and $\left.k_{b}\right)$ and as it exits the outlet $\left(k_{t}\right.$ and $\left.\mu_{\text {int }}\right)$. Increasing the bending elasticity increases cell stiffness and decreases collapse in the tail region of the cell. Decreased external element elasticity allows cells to expand further upon exiting the outlet. Increasing the internal element viscosity slows the rate at which the cell converges to new equilibrium shapes. Relatively little effect was seen when the external element viscosity, $\mu_{\text {ext }}$, was altered.

It is important to highlight the relatively low percentage agreement resulting when using a lower bending elasticity, $k_{b}^{\prime}=k_{b} / 2$. This results because of the physical instability earlier mentioned. The result is not shown here but the instability leads to a small amount of asymmetry as the cell passes through the gap. This asymmetry is relatively quickly amplified so that the cell leaves the gap in an asymmetric shape that relatively quickly carries it upward away from the centerline of the microchannel. This shows that small deviations from expected can be significantly amplified when transported through narrow gaps like those in the microchannel shown here.

Cell shape deviations when the cell passes through the narrow outlet depends mostly on $k_{p}, k_{t}$, and $k_{b}$, which can be thought of as interior and membrane elasticities/rigidities. At the same time, away from the narrow outlet there seems to be a large dependence on $\mu_{\text {int }}$, which can be thought of as the interior viscosity/fluidity. Which of these parameters are more important depends on the situation. For instance, the elasticities may matter more for cell intravasation and extravasation while internal viscosity may matter more for cell translocation. The external viscosity of the membrane does not have a significant impact. The reason for high internal viscosity sensitivity is because after exiting, the elasticity of the cell makes it so that the cell wants to return to its original circular shape. This requires a change in the internal area. This cannot take place without stretching of the internal elements. At the same time, it can take place without stretching the external elements, which is why the external element viscosity does not play as large a role. 

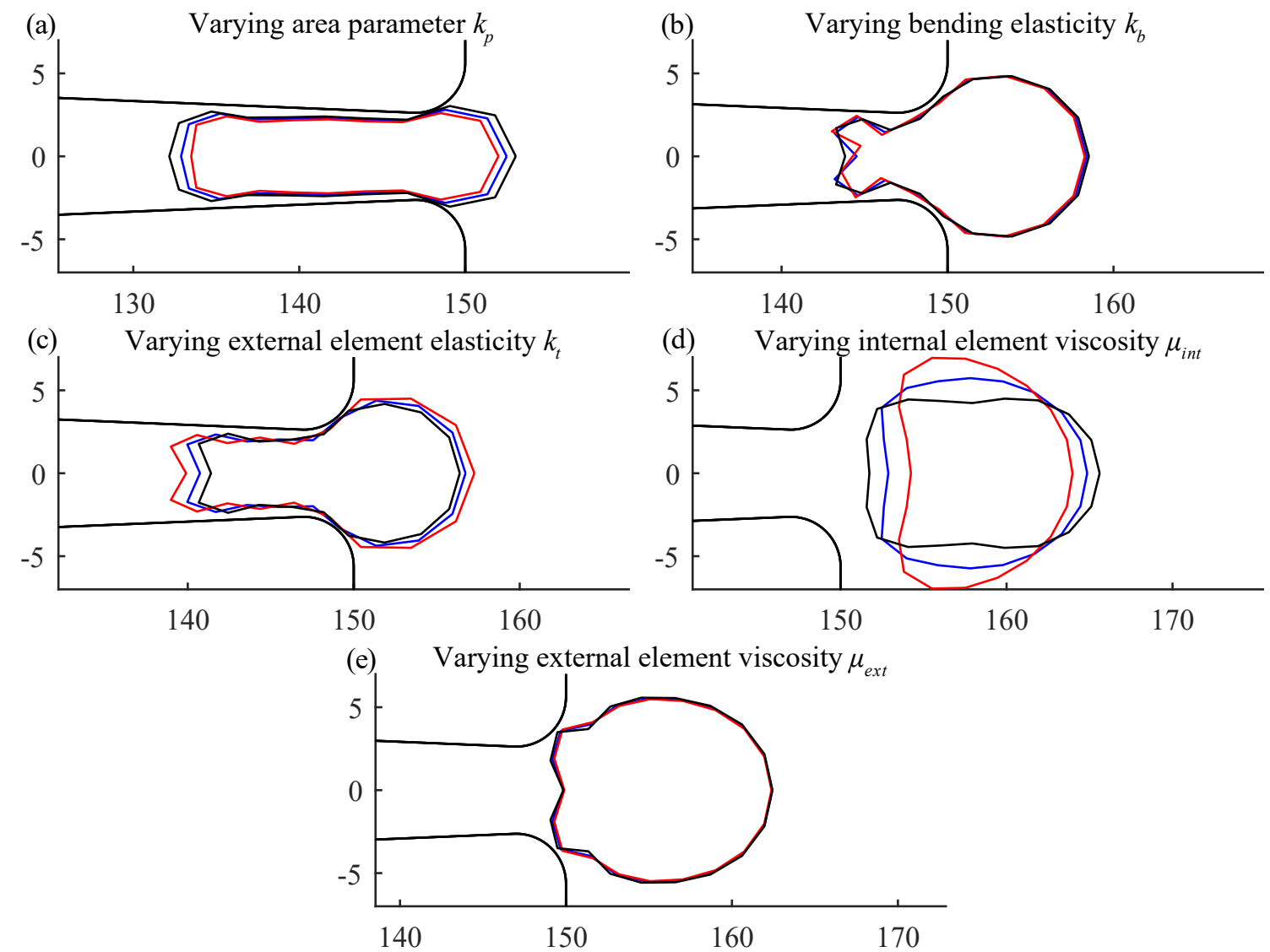

Figure 5: (a) Cell shape comparison for $k_{p}^{\prime}=k_{p} / 2$ (red), $k_{p}$ (blue, baseline), and $k_{p}^{\prime}=2 k_{p}$ (black). (b) Cell shape comparison for $k_{b}^{\prime}=k_{b} / 2$ (red), $k_{b}$ (blue, baseline), and $2 k_{b}$ (black). (c) Cell shape comparison for $k_{t}^{\prime}=k_{t} / 2$ (red), $k_{t}$ (blue, baseline), and $2 k_{t}$ (black). (d) Cell shape comparison for $\mu_{\text {int }}^{\prime}=\mu_{\text {int }} / 2$ (red), $\mu_{\text {int }}$ (blue, baseline), and $2 \mu_{\text {int }}$ (black). (e) Cell shape comparison for $\mu_{\text {ext }}^{\prime}=\mu_{\text {ext }} / 2$ (red), $\mu_{\text {ext }}$ (blue, baseline), and $2 \mu_{\text {ext }}$ (black).

\subsection{Experimental agreement}

With permission from Drs. Hiroki Yokota, Sungsoo Na, and Jong Ryu, Figure 6 shows a simulation alongside experiment. Before parameters were adjusted, cells would fold over upon themselves and elongate or shrink excessively depending upon the scenario and chosen boundary conditions. Now, however, there is relatively good qualitative agreement similar. Still, it can be seen that some calibration remains to be done as the cell in the model is still more elongated than the cell in the experiment. Possible ways to improve the model, including calibration, are discussed below. 
(a)

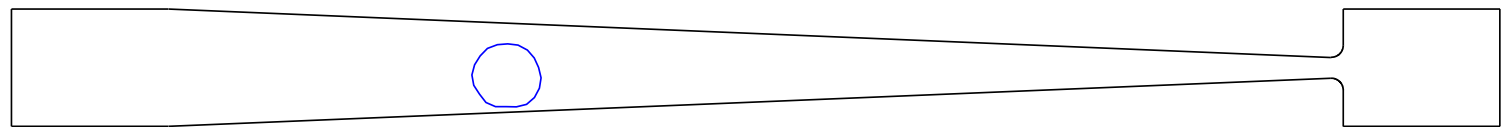

(b)

(c)

Figure 6: Simulation using an off-centered cell compared with experimental pictures at different times. (a) Initial cell shape used for experimental simulation. (b) Shape resulting using baseline parameter values. (c) Shape resulting by introducing elasticity to the internal elements and augmenting the external element elasticity. Model improvements mentioned in the discussion section could help increase agreement.

\section{Discussion}

\subsection{Feasibility study}

The study has shown that the model requires relatively few adjustments in order to produce qualitatively realistic results as well as useful information such as parameter sensitivities, velocity and stress levels, and peak stress locations. This suggests significant potential for the model for use for considering small numbers of cancer cells. This initial feasibility assessment was completed without fully calibrating the model. Calibrations take time and effort including proper consideration of how to either appropriately calibrate a $2 \mathrm{~d}$ model using $3 \mathrm{~d}$ data or how to adapt the $2 \mathrm{~d}$ model to 3 dimensions. An initial feasibility study like this helps determine whether or not such an investment would be worthwhile. Having demonstrated significant promise for this approach, we now plan to fully calibrate the model in order to best match experiments in (TruongVo et al. 2017). The model will then be employed to consider other situations and scenarios including both in vitro and in vivo studies with additional calibration performed on an as-needed basis.

\subsection{Model extensions}

Most simulations presented here have the cell traveling down the center line of the microarray channel. In experiment, cells will not have this exact trajectory but will often be closer to one wall or the other. Despite this, flexible particles tend to migrate away from walls and this combined with the tapered nature of the channel will lead to cell paths that eventually lie very close to the center line. Before that, being nearer to the walls, they will tend to travel slower than cells that start in the middle with simulations. We have not yet quantified the delay time associated with such off-centered cells but plan to especially when starting to consider other microfluidic devices such as vortical sorters.

\subsection{Numerical method}

This method, originally designed for red blood cells, is capable of efficiently producing results qualitatively similar to in vitro results (TruongVo et al. 2017). In particular, while narrow gaps frequently require special attention as in the case of the immersed boundary method (Fai and Rycroft 2018) or a significant investment in computational resources and efficiency (Lykov et al. 2017), this method runs relatively quickly (less than a day per simulation) on personal computing resources (two dedicated cores at $3.2 \mathrm{Ghz}$ ). In addition, the numerical accuracy is quite high with third order accuracy in space, second order in time, and adaptive meshing greatly increasing accuracy in narrow gap regions as in the bottom left of Figure 4. Figure 3 and Figure 4 show the relatively high solution resolution 
produced. While some sacrifices have been made in terms of model accuracy/complexity (see below), the numerical methods are fairly efficient and robust and work well for scenarios where high model accuracy is not necessary.

\subsection{Additional considerations/future work}

4.4.1 Two and three dimensions

To improve the overall model accuracy, a third dimension is desirable. Such a shift will involve a greater number of degrees of freedom making implementation and maintenance of computational efficiency and accuracy more difficult. The overall nature for the system, especially for the microchannel, will also change substantially. For instance, in two dimensions the cell almost completely obstructs the microchannel outlet like a stopper in a drain while in three dimensions, as in the (TruongVo et al. 2017) experiments, cells cannot completely obstruct the channel. Fluid can always flow above or below in order to get around cells. This makes for a less drastic pressure drop across the cell and lower and smoother stresses in general. At the same time, because of this less drastic pressure drop, cells are more likely to become arrested between the two side walls on their way to the channel exit. In fact, in the (TruongVo et al. 2017) experiment this scenario, where cells were prematurely arrested or significantly slowed down, was of primary interest. The current model is not yet capable of considering such situations and it remains to be seen how much the increased difficulty of modeling cell arrestment in three dimensions will be countered by the lowered expected pressure drop and smoother dynamics in the system.

\subsubsection{Model limitations and enhancements}

We have assumed negligible inertial effects. This was based on a relatively low estimated 0.09 Reynolds number obtained using the cell's diameter as a characteristic length and average fluid velocity in the channel. For subcellular components such as the nucleus, we expect the Reynolds number to be even lower because the local characteristic lengths and flow fields will be smaller in magnitude. While inertial effects can be expected to have diminishing importance as the Reynolds number gets smaller, the magnitude of these effects can still be appreciable at Reynolds numbers on the order considered here. In flow dominated by viscous resistance, cell migration towards the center of passageways is primarily affected by cell deformability while in flow where inertial effects are appreciable, cell migration can be affected with cell mass density causing cells with non-negligible momentum to drift more readily across background steady state fluid streamlines. In addition, inertial effects within the cell, such as those associated with the nucleus, could also affect cell motion. We plan to extend our method to consider the full Navier-Stokes equations and understand the importance these cellular and subcellular inertial effects may play in typical cellular dynamics. While our method can be extended, the resulting model will require more computational resources as solving these nonlinear equations (Stokes equations are linear) will take longer.

The cell interior including its nucleus, cytoplasm, various structural proteins, and organelles are currently all lumped together and sparingly modeled using interior viscous elements and an internal pressure. While there is some evidence that a highly detailed model of the cell interior is not necessary for producing useful results (Lykov et al. 2017), one of the advantages of the relatively simple model presented here is that it can (and will) be used to consider how more explicitly modeling cell interior components like the nucleus may or may not affect predicted cell dynamics. Many theorize the nucleus may play a role in circulating tumor cell intravasation and extravasation and understanding its potential role by carefully enhancing the model is of great interest. In addition, a more explicit representation of various cell components can also help improve understanding of the role of the density and type of components included in the cell, which depends on age, cell-type, and other factors.

The cell uses a Kelvin model for each external element, a viscous element for each internal element, and an elasticlike cell interior pressure that resists large cross-sectional area changes. These are attractive because they are both fairly simple models which require no temporal discretization of the tensions in the external element. In addition, other models (Pivkin et al. 2017) have also represented the membrane using viscous elements in parallel with elastic elements suggesting such a model is capable of replicating experiments to some degree. At the same time, it is possible other more complicated viscoelastic models, like the standard linear solid model, may produce more realistic results and this is an avenue we are currently pursuing.

The geometry used includes corners on the cell, which is physiologically unrealistic because cell membranes have relatively smooth surfaces (when inspected at the cellular level, not the subcellular level). There are two ways to address this, use more nodes and/or use curved elements. 20 nodes were used because this number gave reasonable red blood cell results (Barber et al. 2008; Barber et al. 2011) and because stability analysis performed after original parameter selection revealed that use of 40 nodes would require a significant decrease in the time step. Fortunately, 
later sensitivity analysis revealed that the cell shape does not depend significantly on parameters that affect the stability dependent time step. Future work will target adjusting parameter values in such a way that maintains agreement with experiment while maximizing the time step size required when using larger numbers of nodes. Curved elements are also being investigated. A difficulty with such elements is that the tools currently used with this model are not easily modified in order to perform the integrals involving arc lengths in the current formulation of our model. In fact, calculating arc length along many traditional curved interpolants is a non-trivial matter. Nonetheless, not only would use of curved elements improve agreement with the physiology of circulating tumor cells, if properly implemented it will also provide numerical benefits including better behaved flow and stress fields.

The current simulations were slightly adjusted in order to begin considering epithelial (MCF-10A cell line) and cancerous (MDA-MB-231) breast cells as seen in (TruongVo et al. 2017). Further calibration of the model should allow us to work alongside these collaborators in order to help them design microfluidic devices for use in breast cancer cell diagnostics and characterization of cancer cell dynamics in vivo. Calibration will include more explicit comparisons with (TruongVo et al. 2017) data (particularly including cell's that become arrested in the microchannel) and with micropipette experiments like those in (Lykov et al. 2017). We expect that breast cancer cells, which tend to be more flexible, will have lower elastic and viscosity constants in general compared to the normal cells. While mentioning calibration it is important to note that cells differ greatly between their types and that even cancer cells have significantly different characteristics depending upon their place of origin (e.g. breast vs pancreas) and stage in life (Suresh 2007). While theoretically this model could be adapted to many different scenarios with this study suggesting that the model can be somewhat versatile, it must be done carefully.

\section{Conclusions}

With the knowledge that there is rarely a perfect model for every situation, we have explored using a model from the area of red blood cell dynamics to consider dynamics of other cells. This initial feasibility study suggests that this model has strong potential for use in other settings. The model, with strengths in the areas of modeling a low number of cells and resolution in narrow gap regions, is capable of producing reasonable simulations of cells in microchannels. Adjusting this model appropriately for different scenarios, using the model to investigate velocity and stress fields, and performing sensitivity analyses will allow us to more completely characterize the dynamics of breast cancer cells.

\section{Acknowledgements}

This work has been partially supported by the Department of Mathematics in the School of Science at IUPUI, the Biomechanics and Biomaterials Research Center, the Integrated Nanosystems Development Institute, and NSF grant DMS-1522554. We would like to thank H. Yokota and S. Na (bioengineering professors at IUPUI), J. Ryu (mechanical engineering professor at NCSU), and T. TruongVo (research assistant at IUPUI) for discussions regarding their microchannel array experiments and paper. We would also like to thank H. Nakshatri (Marian J Morrison Chair in Breast Cancer Research at IUPUI) for useful discussions on important aspects of breast cancer. Finally, we would like to thank students J. Celaya-Alcala and A. Kovacs who helped us better understand capabilities and limitations of the numerical methods used as well as H. Sledge and C. Bugelholl who helped us investigate the efforts necessary for proper calibration of the model.

Barber JO (2009) Computational simulation of red blood cell motion in microvascular flows. Doctoral dissertation, University of Arizona

Barber JO, Alberding JP, Restrepo JM, Secomb TW (2008) Simulated two-dimensional red blood cell motion, deformation, and partitioning in microvessel bifurcations Ann Biomed Eng 36:1690-1698 doi:10.1007/s10439-008-9546-4

Barber JO, Restrepo JM, Secomb TW (2011) Simulated Red Blood Cell Motion in Microvessel Bifurcations: Effects of Cell-Cell Interactions on Cell Partitioning Cardiovasc Eng Technol 2:349-360 doi:10.1007/s13239-0110064-4

Blumers AL, Tang YH, Li Z, Li XJ, Karniadakis GE (2017) GPU-accelerated red blood cells simulations with transport dissipative particle dynamics Comput Phys Commun 217:171-179 doi:10.1016/j.cpc.2017.03.016

Chin LK, Xia YT, Discher DE, Janmey PA (2016) Mechanotransduction in cancer Curr Opin Chem Eng 11:77-84 doi:10.1016/j.coche.2016.01.011

Crowl LM, Fogelson AL (2010) Computational model of whole blood exhibiting lateral platelet motion induced by red blood cells Int J Numer Meth Bio 26:471-487 doi:10.1002/cnm.1274 
Djukic T, Topalovic M, Filipovic N (2015) Numerical simulation of isolation of cancer cells in a microfluidic chip J Micromech Microeng 25 doi:Artn 084012 10.1088/0960-1317/25/8/084012

Dong C, Skalak R (1992) Leukocyte Deformability - Finite-Element Modeling of Large Viscoelastic Deformation J Theor Biol 158:173-193 doi:Doi 10.1016/S0022-5193(05)80716-7

Drury JL, Dembo M (2001) Aspiration of human neutrophils: Effects of shear thinning and cortical dissipation Biophys J 81:3166-3177 doi:Doi 10.1016/S0006-3495(01)75953-X

Dupin MM, Halliday I, Care CM, Alboul L, Munn LL (2007) Modeling the flow of dense suspensions of deformable particles in three dimensions Phys Rev E 75 doi:Artn 066707 10.1103/Physreve.75.066707

Evans EA, Skalak R (1979) Mechanics and thermodynamics of biomembranes: part 1 CRC Crit Rev Bioeng 3:181330

Fai TG, Rycroft CH (2018) Lubricated immersed boundary method in two dimensions J Comput Phys 356:319-339 doi:10.1016/j.jcp.2017.11.029

Freund JB (2014) Numerical Simulation of Flowing Blood Cells Annu Rev Fluid Mech 46:67-+ doi:10.1146/annurev-fluid-010313-141349

Hoppe RHW, Linsenmann C (2012) An adaptive Newton continuation strategy for the fully implicit finite element immersed boundary method J Comput Phys 231:4676-4693 doi:10.1016/j.jcp.2012.03.004

Hu SH, Liu GY, Chen WQ, Li X, Lu W, Lam RHW, Fu JP (2016) Multiparametric Biomechanical and Biochemical Phenotypic Profiling of Single Cancer Cells Using an Elasticity Microcytometer Small 12:2300-2311 doi:10.1002/smll.201503620

Jaalouk DE, Lammerding J (2009) Mechanotransduction gone awry Nat Rev Mol Cell Bio 10:63-73 doi:10.1038/nrm2597

Jea KC, Young DM (1983) On the simplification of generalized conjugate-gradient methods for nonsymmetrizable linear systems Linear Algebra and its Applications 52-53:399-417 doi:10.1016/0024-3795(83)80026-3

Khodaee F, Movahed S, Fatouraee N, Daneshmand F (2016) Numerical Simulation of Separation of Circulating Tumor Cells from Blood Stream in Deterministic Lateral Displacement (Dld) Microfluidic Channel J Mech 32:463-471 doi:10.1017/jmech.2015.91

Leight JL, Wozniak MA, Chen S, Lynch ML, Chen CS (2012) Matrix rigidity regulates a switch between TGF-beta 1-induced apoptosis and epithelial-mesenchymal transition Mol Biol Cell 23:781-791 doi:10.1091/mbc.E11-06-0537

Leong FY, Li QS, Lim CT, Chiam KH (2011) Modeling cell entry into a micro-channel Biomech Model Mechan 10:755-766 doi:10.1007/s10237-010-0271-1

Lykov K, Nematbakhsh Y, Shang ML, Lim CT, Pivkin IV (2017) Probing eukaryotic cell mechanics via mesoscopic simulations Plos Comput Biol 13 doi:ARTN e1005726 10.1371/journal.pcbi.1005726

Majeski HE, Yang J (2016) The 2016 John J. Abel Award Lecture: Targeting the Mechanical Microenvironment in Cancer Mol Pharmacol 90:744-754 doi:10.1124/mol.116.106765

Marella SV, Udaykumar HS (2004) Computational analysis of the deformability of leukocytes modeled with viscous and elastic structural components Phys Fluids 16:244-264 doi:10.1063/1.1629691

Paredes J, Albergaria A, Oliveira JT, Jeronimo C, Milanezi F, Schmitt FC (2005) P-cadherin overexpression is an indicator of clinical outcome in invasive breast carcinomas and is associated with $\mathrm{CDH} 3$ promoter hypomethylation Clin Cancer Res 11:5869-5877 doi:10.1158/1078-0432.Ccr-05-0059

PDE Solutions Inc (2018) FlexPDE 7: Version 7.08 Documentation. PDE Solutions Inc, Spokane Valley, WA

Peng ZL, Asaro RJ, Zhu Q (2011) Multiscale modelling of erythrocytes in Stokes flow J Fluid Mech 686:299-337 doi: $10.1017 / \mathrm{jfm} .2011 .332$

Peskin CS (1977) Numerical analysis of blood flow in the heart J Comput Phys 25:220-252 doi:10.1016/00219991(77)90100-0

Pivkin IV, Peng ZL, Karniadakis GE, Buffet PA, Dao M, Suresh S (2017) Biomechanics of red blood cells in human spleen and consequences for physiology and disease (vol 113, pg 7804, 2016) P Natl Acad Sci USA 114:E4521-E4521 doi:10.1073/pnas.1706577114

Pozrikidis C (2005) Numerical simulation of cell motion in tube flow Ann Biomed Eng 33:165-178 doi:10.1007/s10439-005-8975-6

Rejniak KA (2012) Investigating dynamical deformations of tumor cells in circulation: predictions from a theoretical model Front Oncol 2:111 doi:10.3389/fonc.2012.00111

Rejniak KA (2016) Circulating Tumor Cells: When a Solid Tumor Meets a Fluid Microenvironment. In: Rejniak KA (ed) Systems Biology of Tumor Microenvironment. Springer International Publishing, Switzerland, pp 93-106 
Ribeiro AS et al. (2010) Co-expression of E- and P-cadherin in breast cancer: role as an invasion suppressor or as an invasion promoter? Ejc Suppl 8:131-132 doi:Doi 10.1016/S1359-6349(10)71315-X

Secomb TW (1987) Flow-dependent rheological properties of blood in capillaries Microvasc Res 34:46-58

Secomb TW, Styp-Rekowska B, Pries AR (2007) Two-dimensional simulation of red blood cell deformation and lateral migration in microvessels Ann Biomed Eng 35:755-765 doi:10.1007/s10439-007-9275-0

Suresh S (2007) Biomechanics and biophysics of cancer cells Acta Biomater 3:413-438 doi:10.1016/j.actbio.2007.04.002

Swaminathan V, Mythreye K, O'Brien ET, Berchuck A, Blobe GC, Superfine R (2011) Mechanical Stiffness Grades Metastatic Potential in Patient Tumor Cells and in Cancer Cell Lines Cancer Res 71:5075-5080 doi:10.1158/0008-5472.Can-11-0247

Tremblay PL, Huot J, Auger FA (2008) Mechanisms by which E-selectin regulates diapedesis of colon cancer cells under flow conditions Cancer Res 68:5167-5176 doi:10.1158/0008-5472.CAN-08-1229

TruongVo T et al. (2017) Microfluidic channel for characterizing normal and breast cancer cells J Micromech Microeng 27:035017 